\title{
Mechanisims of asthma and allergic disease - 1093. A novel human anti-VCAM-1 Monoclonal antibody Ameliorates airway inflammation and remodeling in murine asthma model
}

\author{
Jae-Hyun Lee', Jung-Ho Sohn², Su Yeon Ryu ${ }^{3}$, Kyung D Moon ${ }^{3}$, Chein-Soo Hong ${ }^{1}$, Jung-Won Park ${ }^{\text {1* }}$ \\ From 2nd WAO International Scientific Conference (WISC 2012) \\ Hyderabad, India. 6-9 December 2012
}

\section{Background}

Asthma is a chronic inflammatory disease induced by Type 2helper T cells (Th2) and eosinophils. Vascular cell adhesion molecule-1(VCAM-1) is the regulatory receptor implicated with recruiting eosinophils andlymphocytes to pathologic site in asthma. A monoclonal antibody (mAb)against VCAM-1 may attenuate allergic inflammation and pathophysiologicfeatures of asthma. Weevaluated whether a recently developed human antiVCAM-1mAb can inhibit pathophysiologic features of asthma in a murine asthma modelinduced by ovalbumin (OVA).

\section{Methods}

We evaluated whether human anti-VCAM-1 mAb binds to human ormouse VCAM-1. Leukocyte adhesion inhibition assay was performed toevaluate the in vitro blocking activity of human anti-VCAM-1 mAb. OVAsensitized $\mathrm{BALB} / \mathrm{c}$ mice were treated with human anti-VCAM-1 mAb orisotype control Ab before intranasal OVA challenge. We evaluated airwayhyperresponsiveness (AHR) and cell counts in bronchoalveolar lavage (BAL)fluid, measured inflammatory cytokines, and examined histopathologicalfeatures, including VCAM-1 immunohistochemistry.

\section{Results}

The human anti-VCAM-1 mAb bound to human and mouse VCAM-1molecules and inhibited adhesion of human leukocytes in vitro. AHR andinflammatory cell counts in BAL fluid were reduced in mice treated withhuman anti-VCAM-1 mAb as compared to a control Ab. The levels ofinterleukin (IL)-5 and IL-13, and transforming growth factor $-\beta$ in lung tissuewere decreased in treated mice. Human anti-VCAM-1 mAb reduced goblet cellhyperplasia and peribronchial fibrosis. In vivo VCAM-1 expression decreasedin treated group.

\section{Results}

Human anti-VCAM-1 mAb can attenuate allergic inflammationand pathophysiological features of asthma in OVA induced murine asthmamodel. This data suggested that human anti-VCAM-1 mAb could be an additionalantiasthma therapeutic medicine.

\section{Author details}

'Div. of Allergy and Immunology, Dept. of Internal Medicine, Yonsei University College of Medicine, Seoul, South Korea. ${ }^{2}$ Department of Life Science, Research Institute for Natural Sciences, Hanyang University, Seoul, South Korea. ${ }^{3}$ Hanwha Chemical R\&D Center, Daejeon, South Korea.

\section{Published: 23 April 2013}

\section{doi:10.1186/1939-4551-6-S1-P89}

Cite this article as: Lee et al.: Mechanisims of asthma and allergic disease - 1093. A novel human anti-VCAM-1 Monoclonal antibody Ameliorates airway inflammation and remodeling in murine asthma model. World Allergy Organization Journal 2013 6(Suppl 1):P89. 